\title{
Comparison of Conventional and Inverted Organic Photovoltaic Devices with Controlled Illumination Area and Extraction Layers
}

Yun-Ju Lee, ${ }^{1}$ Brandon L. Adkison, ${ }^{2}$ Liang Xu, ${ }^{1}$ Aaron A. Kramer, ${ }^{3}$ and Julia W. P. Hsu ${ }^{1}$

Dr. Y.-J. Lee, L. Xu, Prof. J. W. P. Hsu

Department of Materials Science and Engineering, University of Texas at Dallas

800 W. Campbell Rd., Richardson, TX 75080 USA

B. L. Adkison

Department of Biochemical Engineering, University of Texas at Dallas

800 W. Campbell Rd., Richardson, TX 75080 USA

A. A. Kramer

Department of Physics, Tarleton State University

1333 W. Washington Street, Stephenville, TX 76401 USA

Email: jwhsu@utdallas.edu

Keywords: excitonic solar cells, shadow mask, unintentional doping, organic semiconductors, drift-diffusion simulation

The impact of device architecture on organic photovoltaic device performance has been problematic to quantify due to extraction layer materials and device testing issues. In particular, published reports have used different pairs of hole and electron extraction layer materials for conventional versus inverted devices. The origins of the large apparent discrepancy in device J-V characteristics become difficult to understand, arising from differences in built-in potentials and in the extraction layers' conductivities that can affect collection of stray current outside of device 
area. Here, we show that by using an identical pair of hole and electron extraction layers, and by precisely defining illuminated device area with a shadow mask, most of the differences in the performance of conventional and inverted P3HT:PCBM organic photovoltaic devices are eliminated. The remaining difference in short-circuit current density and EQE spectral shape can be explained primarily by the ratio of hole and electron mobility that leads to more efficient carrier extraction in the inverted devices, with a minor contribution from unintentional p-type doping of the active layer that influences the drift field profile. 


\section{Introduction}

Organic photovoltaics (OPVs) have generated significant research interest over the past decade due to the promise of low-cost, lightweight renewable energy generation $[1,2]$. However, accurate measurements of the power conversion efficiency (PCE) of OPVs have been complicated by various factors, including spectral mismatch between light source and reference photodiode [3], stray current due to high conductivity transport layers [4-7], and unintentional absorption of reflected or scattered light [7-9]. The uncontrolled illumination and variation in ambient temperature and humidity can be minimized through the use of enclosed sample holders.[10-12] The device architecture itself may also affect PCE. Extraction layers (ELs) are often inserted between the active layer and the electrodes of OPVs to determine polarity and improve performance $[13,14]$. OPVs with conventional versus inverted architectures, where the holes collected by the front versus back electrode, have been shown to exhibit discrepancy in performance that have been attributed to vertical phase separation during active layer drying [1517], mismatched electron and hole mobility in the active layer [18-21], resistive losses between different ELs and electrode [22], and unwanted absorption of incident light by the EL [23-25]. Furthermore, even direct experimental comparisons of conventional versus inverted OPVs utilize different pairs of ELs [20,22,23], due to the competing requirements of optical transparency, electrical conductivity, wettability, and chemical miscibility between subsequent layers, and mild deposition conditions to maintain active layer nanostructure. As a result, the two device architectures may also exhibit significant differences in e.g. built-in potential and EL conductivities, and the observed differences in $\mathrm{J}-\mathrm{V}$ response may be a combination of active layer and EL effects. In this report, we present a quantitative experimental-computational study of the effect of device architecture on poly(3-hexylthiophene): [6,6]-phenyl-C61-butyric acid 
methyl ester (P3HT:PCBM) OPV performance. In particular, we use, for the first time, an identical pair of transparent metal oxides as ELs for conventional and inverted devices, and combine them with a shadow mask to explicitly define illumination area, hence eliminating any discrepancy due to stray current [8]. While most device performance parameters become equal for the two architectures, differences beyond experimental uncertainties remain in short-circuit current density and the external quantum efficiency (EQE) spectra. After examining built-in potential and light absorption and performing drift-diffusion modeling with varying doping density and carrier mobility, we find that the observed discrepancy is primarily due to the ratio of hole and electron mobility that leads to more efficient carrier extraction in inverted devices, with a minor contribution from unintentional p-type doping of the active layer that affects the electric field profile inside the device.

\section{Materials and Methods}

OPVs fabrication. Conventional and inverted P3HT:PCBM OPVs were fabricated following published procedure [26] with modifications. All chemicals were used as received, and all processing was performed in a $\mathrm{N}_{2}$ glove box (Innovative Technology) unless otherwise noted. For conventional devices, $10 \mathrm{~nm} \mathrm{MoO} 3(99.999 \%$ Alfa Aesar) was thermally evaporated (Angstrom Engineering) on patterned ITO on glass ( $20 \Omega / \square$, Thin Film Devices) that has been cleaned with UV-ozone (ProCleaner Plus, BioForce) for $20 \mathrm{~min}$ to form the hole EL (HEL). P3HT (RMI-001E, Rieke) and $\mathrm{PC}_{71} \mathrm{BM}$ (Solenne BV) were dissolved at 23:23 mg/mL in chlorobenzene (Aldrich) and spin coated at $1200 \mathrm{rpm}$ to form $250 \mathrm{~nm}$ thick BHJ. A suspension of $\mathrm{ZnO}$ nanocrystals in n-butanol was synthesized [26] and spin coated at $4000 \mathrm{rpm}$ twice to form the electron EL (EEL) on top of the active layer. The sample was then annealed in $\mathrm{N}_{2}$ at $170{ }^{\circ} \mathrm{C}$ for $10 \mathrm{~min}$. Finally, $100 \mathrm{~nm} \mathrm{Ag}$ was thermally evaporated to complete the OPVs. For 
inverted OPVs, $\mathrm{ZnO}$ nanocrystals and P3HT:PCBM were subsequently spin coated on patterned ITO at the previously noted conditions, and the sample was annealed in $\mathrm{N}_{2}$ at $170{ }^{\circ} \mathrm{C}$ for $10 \mathrm{~min}$. $10 \mathrm{~nm} \mathrm{MoO}$ and $100 \mathrm{~nm} \mathrm{Ag}$ layers were thermally evaporated on to complete the OPVs. The electrode intersection area is $0.11 \mathrm{~cm}^{2}(3.2 \mathrm{~mm}$ ITO x $3.6 \mathrm{~mm} \mathrm{Ag})$. OPVs characterization. The light source was a class AAA solar simulator (Sun 3000, Abet Technologies) with AM1.5G filter, at intensity of $100 \mathrm{~mW} \mathrm{~cm}^{-2}$ set using a calibrated $\mathrm{Si}$ photodiode with KG5 filter (RR_227 KG5, Abet Technologies). The sample holder was a modified anodized aluminum KF50 vacuum flange, as previously reported (Fig. 1a) [11]. The illumination area was further limited to $0.049 \mathrm{~cm}^{2}$ by inserting a shadow mask consisting of a 150 um thick stainless steel film with $0.25 \mathrm{~cm}$ diameter laser cut holes, inserted between the sample holder and the device. Two aperture geometries were tested: one with six apertures aligned to the six devices (" 6 ap", Fig. 1b), and one with only a single aperture aligned to one device at a time ("1 ap", Fig. 1c). J-V measurements were performed in $\mathrm{N}_{2}$ using a low-noise sourcemeter (2635A, Keithley) controlled by LabView (National Instruments). All data shown represent the average of at least six devices. External quantum efficiency (EQE) spectra was collected at room temperature under one-sun white-light bias using a homemade system [21]. Capacitance-voltage $(\mathrm{C}-\mathrm{V})$ measurements were carried out at room temperature in dark using a Zahner IM6 electrochemical workstation. Varying AC modulation frequency (10Hz to $1 \mathrm{MHz})$ and DC bias (-1 to $0.5 \mathrm{~V}$ ) were used, while the amplitude of AC modulation was fixed at $20 \mathrm{mV}$. Capacitance was directly calculated from the imaginary component of the complex impedance at $1 \mathrm{kHz}$ assuming negligible inductance. 
OPVs simulation. Simulation of carrier generation inside the active layer was calculated using the transfer matrix method (TMM) implemented in MATLAB (MathWorks) [27]. $\mathrm{n}, \mathrm{k}$, and thickness of each OPV layer (ITO, P3HT:PCBM, $\mathrm{MoO}_{3} \mathrm{ZnO}$ nanocrystal) on Si wafer were obtained form spectroscopic ellipsometry (M-2000DI, J. A. Woollam) data fitted to a built-in model ("B-Spline"). Optical absorption as a function of position within the P3HT:PCBM layer was calculated using a layer stack of ITO $(140 \mathrm{~nm}) / \mathrm{MoO}_{3}(10 \mathrm{~nm}) / \mathrm{P} 3 \mathrm{HT}: \mathrm{PCBM}(250 \mathrm{~nm}) / \mathrm{ZnO}$ nanocrystals $(30 \mathrm{~nm}) / \mathrm{Ag}(100 \mathrm{~nm})$ for conventional devices and ITO $(140 \mathrm{~nm}) / \mathrm{ZnO}$ nanocrystals $(30 \mathrm{~nm}) / \mathrm{P} 3 \mathrm{HT}: \mathrm{PCBM}(250 \mathrm{~nm}) / \mathrm{MoO}_{3}(10 \mathrm{~nm}) / \mathrm{Ag}(100 \mathrm{~nm})$ for inverted devices. A profile of generation rate versus position in active layer was calculated for each wavelength between 400 $\mathrm{nm}$ and $700 \mathrm{~nm}$ at $10 \mathrm{~nm}$ increments by integrating the absorption data with $100 \mathrm{~mW} \mathrm{~cm}$ AM1.5G spectral intensity. 1-D drift-diffusion simulation of OPVs was performed using SCAPS [28] to calculate $\mathrm{J}-\mathrm{V}$ curves and band diagrams of conventional and inverted devices, using total generation profiles calculated by TMM as input and assuming bimolecular recombination. Wavelength dependent generation profiles calculated by TMM were used to simulate EQE spectra.

\section{Results and Discussion}

Fig. 2 shows the J-V data of conventional and inverted OPVs under three illumination conditions: with no shadow mask, with 6 ap mask, and with 1 ap mask. The mask dimensions are ideal according to the recommendations from Gevorgyan and co-workers, who systematically studied the effect of shadow mask geometry on measured performance of OPVs and found that an opaque mask adjacent to the device, with a thickness of $<0.4 \mathrm{~mm}$ and aperture size at least $0.5 \mathrm{~mm}$ smaller than electrode size, minimized the effect of solar simulator beam divergence and yielded high accuracy in measurement of OPV performance [8]. However, Gevorgyan and co- 
workers only studied single apertures centered on individual devices, i.e. similar to our 1 ap mask. Because OPV sample typically contain multiple devices per substrate, the shadow mask would need to be repositioned between measurements of different devices, which significantly increase measurement time and complexity. Thus, we compare OPVs with 6 ap mask versus 1 ap mask to determine the impact, if any, on device parameters. We find that for conventional OPVs, a change from no mask (Fig. 2a, dotted) to 6 ap mask (Fig. 2a, dashed) decreases short circuit current density $\mathrm{J}_{\mathrm{sc}}$ from 9.66 to $9.04 \mathrm{~mA} \mathrm{~cm}^{-2}$, while increasing factor $\mathrm{FF}$ from 0.576 to 0.591 (Table 1). Further changing to 1 ap mask (Fig. 2a, solid) does not modify $\mathrm{J}_{\mathrm{sc}}$ or FF. When the performance parameters are normalized to the no mask condition, we see that application of either 6 ap mask or 1 ap mask causes a $7 \%$ decrease in $\mathrm{J}_{\mathrm{sc}}$, compensated by a $3 \%$ increase in $\mathrm{FF}$ (Fig. 2b). For inverted devices, a change from no mask (Fig. 2c, dotted) to 6 ap mask (Fig. 2c, dashed) decreases $\mathrm{J}_{\mathrm{sc}}$ from 10.99 to $10.11 \mathrm{~mA} \mathrm{~cm}^{-2}$ and increases $\mathrm{FF}$ from 0.533 to 0.565 (Table 1). Unlike the conventional devices, changing to 1 ap mask (Fig. 2c, solid) further decreases $J_{s c}$ to $9.69 \mathrm{~mA} \mathrm{~cm}^{-2}$ and increases $\mathrm{FF}$ to 0.576 (Table 1), which represents a $12 \%$ decrease and $8 \%$ increase respectively compared to the no mask condition (Fig. 2d). A decrease in $\mathrm{J}_{\mathrm{sc}}$ and increase in FF with more restrictive illumination conditions agree with suppressed collection of stray current arising from absorption outside the electrode overlapping region.

Despite using identical EL pair and illumination conditions (1 ap mask), a difference is still observed between conventional and inverted OPVs, particularly the $6 \%$ higher $\mathrm{J}_{\mathrm{sc}}$ of the inverted devices (Table 1). When we integrate the EQE response with $100 \mathrm{~mW} \mathrm{~cm}^{-2} \mathrm{AM} 1.5 \mathrm{G}$ spectral intensity, we find that the inverted OPV exhibits $5 \%$ higher $\mathrm{J}_{\mathrm{sc}}$ compared to the conventional $\mathrm{OPV}$, which is reasonable given that the EQE illumination spot is a focused beam with diameter of $\sim 1 \mathrm{~mm}$, most similar to 1 ap mask. In addition, when EQE spectra of conventional and 
inverted OPVs are compared, the inverted OPV (Fig. 3a, red) displays higher EQE response between $450 \mathrm{~nm}$ and $600 \mathrm{~nm}$ compared to the conventional OPV (Fig. 3a, blue).

To understand this difference, we first examine the quasi-flat band potential $\mathrm{V}_{\text {pos }}$ for the devices, which corresponds to the voltage at which the photocurrent density $\mathrm{J}_{\mathrm{ph}}=\mathrm{J}_{\text {light }}-\mathrm{J}_{\text {dark }}$ contains an inflection point [29]. At $V_{\text {pos }}$, the majority of the active layer experiences zero electric field. Due to the significant band bending at the interface of electrodes and organic active layer, $\mathrm{V}_{\mathrm{pos}}$ is a more accurate measure of the built-in potential across the active layer than the work function difference between the contacts or the voltage determined from Mott-Schottky analysis (Fig. 5a, arrow) [29]. When we plot $d J_{p h} / d V$ versus $V$ and located the maximum (Fig. 3b), we find that $\mathrm{V}_{\text {pos }}$ is $(0.55 \pm 0.01) \mathrm{V}$ for all devices independent of conventional or inverted architecture, consistent with the use of identical EL pair. Thus, a difference in built-in potential is not responsible for the difference in $\mathrm{J}_{\mathrm{sc}}$ between conventional and inverted OPVs. We then calculate the fractional absorption spectra for the P3HT:PCBM active layer using TMM (Fig. 4a), which shows that the conventional OPV exhibits the same general spectral shape compared to the inverted OPV, but with higher absorption between 400 and $600 \mathrm{~nm}$, resulting in a $4 \%$ higher generation current in the conventional OPV when integrated with AM1.5G spectral intensity. In other words, absorption of light cannot explain the higher experimental $\mathrm{J}_{\mathrm{sc}}$ or the enhanced EQE response between $450 \mathrm{~nm}$ and $600 \mathrm{~nm}$ for the inverted OPV. From the curves in Fig. 4a, we derive the normalized cumulative generation as a function of position in the active layer by adding generation rates up to the position and dividing by the total generation rate (Fig. $4 b)$. It is clear that the majority of carriers are generated within $100 \mathrm{~nm}$ of the front electrode for both conventional and inverted OPVs. 
To fully understand the effect of possible carrier loss during extraction on device performance, we perform drift-diffusion simulation of conventional and inverted OPVs using SCAPS, with the material parameters summarized in Table 2. To enable convergence of SCAPS simulation, the active layer/ZnO EEL interface is approximated as a metallic contact with hole recombination velocity of $1 \times 10^{-5} \mathrm{~cm} / \mathrm{s}$. Where possible, the material parameters are measured in our laboratory. For example, we determine the acceptor dopant concentration $\mathrm{N}_{\mathrm{A}}$ of the P3HT:PCBM active layer from C-V measurements. Since P3HT is a p-type semiconductor [30], the cathode forms a Schottky contact with the P3HT:PCBM BHJ and a corresponding depletion region. Therefore, $\mathrm{N}_{\mathrm{A}}$ of the active layer can be determined from the bias dependent depletion layer capacitance [31,32]:

$N_{A}=\frac{2}{q \varepsilon A^{2}}\left(\frac{1}{d\left(C^{-2}\right) / d V}\right)$

where $\mathrm{q}$ is elemental charge, $\varepsilon$ is dielectric permittivity, $\mathrm{A}$ is device area, $\mathrm{C}$ is capacitance, and $\mathrm{V}$ is applied bias. Fig. 5a illustrates the Mott-Schottky plot of a conventional OPV with capacitance measured at $1 \mathrm{kHz}$ from impedance spectroscopy. A straight line is fitted between $-0.5 \mathrm{~V}$ and 0.3 $\mathrm{V}$, from which $\mathrm{N}_{\mathrm{A}}$ of $7 \times 10^{15} \mathrm{~cm}^{-3}$ is obtained. An inverted OPV gives similar results. J-V curves of hole-only devices and electron-only devices are used to extract mobilities [33]: we obtain a hole mobility $\mu_{\mathrm{h}}$ of $5 \times 10^{-4} \mathrm{~cm}^{2} \mathrm{~V}^{-1} \mathrm{~s}^{-1}$ and an electron mobility $\mu_{\mathrm{e}}$ of $2 \times 10^{-3} \mathrm{~cm}^{2} \mathrm{~V}^{-1} \mathrm{~s}^{-1}$ (Supplemental Data, Fig. S1). Drift-diffusion simulation using these material parameters shows that the conventional and inverted OPVs exhibit similar $\mathrm{V}_{\mathrm{oc}}$ and FF (Table 3), but the inverted device shows a $2 \%$ higher $\mathrm{J}_{\mathrm{sc}}$ compared to the conventional device, in good agreement with the experimental data. We note again that the higher $\mathrm{J}_{\mathrm{sc}}$ for inverted OPVs is unexpected, given that the inverted architecture fosters lower absorption of incident light according to TMM 
calculations. Thus, drift-diffusion simulation clearly shows that photogenerated carriers in conventional OPV suffer greater recombination loss during extraction. In addition, the simulated EQE spectra for inverted OPV display higher response between $450 \mathrm{~nm}$ and $600 \mathrm{~nm}$ compared to conventional OPV (Fig. 5b), exhibiting the same trend as the experimental data.

Additional drift-diffusion simulations are performed to pinpoint the underlying cause for the differences in $\mathrm{J}_{\mathrm{sc}}$ and $\mathrm{EQE}$ shape. It has been reported previously that high levels of p-type doping in the active layer can lead to asymmetric band bending such that the depletion is confined to the EEL/cathode [34-39], which can change the shape of the EQE spectra [38]. However, simulated band diagrams for conventional (Fig. 5c, blue) and inverted (Fig. 5c, red) OPVs at short circuit show that for $\mathrm{N}_{\mathrm{A}}=7 \times 10^{15} \mathrm{~cm}^{-3}$, the depletion region extends through the entire active layer, with drift field magnitude greater than $1 \times 10^{4} \mathrm{~V} \mathrm{~cm}^{-1}$ (Fig. 5d). We find that $\mathrm{N}_{\mathrm{A}}$ must be increased to $4 \times 10^{16} \mathrm{~cm}^{-3}$ for both conventional and inverted OPVs to exhibit asymmetric band bending similar to published results for OPVs with different donors [36,38,39], with a field-free region near the HEL and a high-field region near the EEL. Simulated $\mathrm{J}_{\mathrm{sc}}$ values as a function of $\mathrm{N}_{\mathrm{A}}$ (Fig. 6a) show that inverted device exhibit significantly higher $\mathrm{J}_{\mathrm{sc}}(>20 \%)$ over conventional device only when $\mathrm{N}_{\mathrm{A}} \geq 4 \times 10^{16} \mathrm{~cm}^{-3}$, i.e. above the asymmetric band bending threshold. This is consistent with the fact that the high field and high carrier generation regions overlap in inverted OPV but are offset in conventional OPV, resulting in higher carrier extraction efficiency in inverted OPV. On the low $\mathrm{N}_{\mathrm{A}}$ range $\left(\leq 2 \times 10^{15} \mathrm{~cm}^{-3}\right)$, simulation shows that $\mathrm{J}_{\mathrm{sc}}$ of conventional OPV is slightly higher than that of inverted OPV, as would be expected from the higher absorption of incident light (Fig. 4b); in this case, the potential drops uniformly as a function of distance from the HEL for both device architectures. At our experimental $\mathrm{N}_{\mathrm{A}}$ value of $7 \times 10^{15} \mathrm{~cm}^{-3}$ (line in Fig. 6a), while the p-type doping is insufficient to create a field-free region, 
it does contribute to additional band bending near the EEL for both device architectures (Fig. 5c). As a result, the inverted OPV exhibits higher drift field magnitude within $100 \mathrm{~nm}$ of the front EL compared to the conventional OPV (Fig. $5 \mathrm{~d}$ ), and qualitatively explains the higher $\mathrm{J}_{\mathrm{sc}}$. Nevertheless, while band bending becomes asymmetric as $\mathrm{N}_{\mathrm{A}}$ increases, the effect is small at our experimentally determined $\mathrm{N}_{\mathrm{A}}$ value. We also varied bimolecular recombination coefficient $\mathrm{k}$ using drift-diffusion simulation, but found that it has minimal effect on $\mathrm{J}_{\mathrm{sc}}$ for both inverted and conventional OPVs near the experimentally measured k value (Supplementary Data, Fig. S2). Thus, another factor must play a dominant role in determining the efficiency of carrier extraction.

We then vary $\mu_{\mathrm{h}}$ while holding electron mobility $\mu_{\mathrm{e}}$ at $2 \times 10^{-3} \mathrm{~cm}^{2} \mathrm{~V}^{-1} \mathrm{~s}^{-1}$, which mimics the real behavior for P3HT:PCBM active layer, where $\mu_{\mathrm{h}}$ depends strongly P3HT crystallinity and $\mu_{\mathrm{e}}$ is relatively insensitive to processing [40]. We find that changes in $\mu_{\mathrm{h}}$ have a large and nonmonotonic effect on $\mathrm{J}_{\mathrm{sc}}$ due to accumulation of unextracted carriers. As $\mu_{\mathrm{h}}$ increases, a shorter time is required for holes to reach the anode and be extracted. As a result, the active layer shifts from an accumulation of holes at low $\mu_{\mathrm{h}}$ to an accumulation of electrons at high $\mu_{\mathrm{h}}$, both of which screens the built-in field [40,41]. An optimal $\mu_{\mathrm{h}}$ value should exist where balanced carrier extraction minimizes accumulation of both electrons and holes, and maximizes $\mathrm{J}_{\mathrm{sc}}$. Indeed, a plot of $\mathrm{J}_{\mathrm{sc}}$ as a function of $\mu_{\mathrm{h}}$ (Fig. 6b) shows maxima for both device architectures with the corresponding optimal $\mu_{\mathrm{h}}$ value being higher for inverted OPV $\left(3 \times 10^{-4} \mathrm{~cm}^{2} \mathrm{~V}^{-1} \mathrm{~s}^{-1}\right)$ than for conventional OPV $\left(1.5 \times 10^{-4} \mathrm{~cm}^{2} \mathrm{~V}^{-1} \mathrm{~s}^{-1}\right)$. The difference in optimal $\mu_{\mathrm{h}}$ value is due to the fact that holes in inverted OPV have to travel a greater distance to reach the HEL/anode since the majority of carriers are generated near the front electrode (Fig. 4b). Thus, it is understandable that a higher $\mu_{\mathrm{h}}$ is needed to maintain balanced carrier extraction in inverted OPVs. At the 
experimental $\mu_{\mathrm{h}}$ of $5 \times 10^{-4} \mathrm{~cm}^{2} \mathrm{~V}^{-1} \mathrm{~s}^{-1}$, carrier extraction is relatively balanced, particularly for the inverted OPV. This can be seen in the bimolecular recombination rate profile for the inverted OPV (Fig. 6d, solid). Because bimolecular recombination rate depends on the product of hole and electron concentrations, a flat recombination rate profile away from the contacts indicates that neither type of carriers is accumulating in the bulk of the active layer. A decrease in $\mu_{\mathrm{h}}$ to $1 \mathrm{x}$ $10^{-4} \mathrm{~cm}^{2} \mathrm{~V}^{-1} \mathrm{~s}^{-1}$ caused the inverted OPV to exhibit $14 \%$ lower $\mathrm{J}_{\mathrm{sc}}$ (Fig. 6b). The corresponding recombination rate profiles show that for both conventional (Fig. 6c, dotted) and inverted (Fig. 6d, dotted) OPVs, recombination rate near the EEL significantly increases due to accumulation of unextracted holes in the front of the inverted device and in the rear of the conventional device. Because the high recombination and the high carrier generation regions overlap in the inverted OPV but are offset in the conventional OPV, carrier extraction and $\mathrm{J}_{\mathrm{sc}}$ decrease more in the inverted device. On the other hand, an increase in $\mu_{\mathrm{h}}$ to $2 \times 10^{-3} \mathrm{~cm}^{2} \mathrm{~V}^{-1} \mathrm{~s}^{-1}$ caused the conventional OPV to exhibit $6 \%$ lower $\mathrm{J}_{\mathrm{sc}}$ (Fig. 6b). In this case, recombination rate near the HEL increases for both conventional (Fig. 6c, dot dash) and inverted (Fig. 6d, dot dash) OPVs from fast extraction of holes and resulting accumulation of electrons. Due to the overlap of the high recombination and high carrier generation regions in the conventional OPV, carrier extraction and $\mathrm{J}_{\mathrm{sc}}$ decrease more in the conventional device.

Comparing Figs. $6 \mathrm{a}$ and $6 \mathrm{~b}$, it is clear that $\mathrm{J}_{\mathrm{sc}}$ depends more strongly on $\mu_{\mathrm{h}}$ than on $\mathrm{N}_{\mathrm{A}}$, particularly near the experimentally measured values for typical devices. We should note that drift-diffusion simulation presents a simplified model of carrier transport in OPVs, since it assumes the bulk heterojunction as a homogenous medium, with no microscopic information on the donor/acceptor morphology and a single bimolecular recombination coefficient. Thus, one cannot expect perfect matching between simulated and experimental results. Nevertheless, drift- 
diffusion simulation allows us to vary individual material parameters and establish their relative impact on measured device properties. In summary, drift-diffusion simulation gives good agreement with experimental data in showing a higher $\mathrm{J}_{\mathrm{sc}}$ and enhanced $\mathrm{EQE}$ response between $450 \mathrm{~nm}$ and $600 \mathrm{~nm}$ for inverted versus conventional OPVs. Moreover, $\mathrm{J}_{\mathrm{sc}}$ is found to depend strongly on hole mobility and moderately on dopant concentration near the experimentally measured values, suggesting that ratio of hole to electron mobility primarily determines the performance discrepancy for the two device architectures.

\section{Conclusions}

We find differences beyond experimental uncertainties for performance of P3HT:PCBM OPVs of conventional and inverted architectures with identical ELs under controlled illumination conditions that eliminate stray photocurrent contributions. The differences are examined using transfer matrix method and drift-diffusion simulation. The higher $\mathrm{J}_{\mathrm{sc}}$ and enhanced $\mathrm{EQE}$ response between $450 \mathrm{~nm}$ and $600 \mathrm{~nm}$ for inverted OPVs are successfully reproduced using drift-diffusion simulation. Systematic variations of material parameters used for simulation reveal that the experimentally observed performance difference between the two architectures results primarily from carrier mobilities that are serendipitously optimized in the case of inverted OPVs, with a minor contribution of band bending due to unintentional p-type doping of the active layer. We show that an approach which combines accurate measurements of OPV performance and careful numerical simulations of carrier generation and recombination is essential to explain and predict the response of OPVs with different active layer and EL materials, as well as other solar cells.

\section{Acknowledgements}

We thank Prof. R. Wallace for the use of the spectroscopic ellipsometer and T. Daunis for calculating the generation profiles using TMM. This research is sponsored by National Science 
Foundation (NSF) DMR-1305893. J.W.P.H. acknowledges the support from Texas Instruments Distinguished Chair in Nanoelectronics. 


\begin{tabular}{lllll}
\hline Device & $\begin{array}{l}\mathrm{V}_{\text {oc }} \\
{[\mathrm{V}]}\end{array}$ & $\begin{array}{l}\mathrm{J}_{\mathrm{sc}} \\
{\left[\mathrm{mA} \mathrm{cm}^{-2}\right]}\end{array}$ & FF & $\begin{array}{l}\text { PCE } \\
{[\%]}\end{array}$ \\
\hline Conv. no mask & $0.558 \pm 0.004$ & $9.66 \pm 0.17$ & $0.576 \pm 0.018$ & $3.11 \pm 0.13$ \\
Conv. 6 ap & $0.559 \pm 0.005$ & $9.04 \pm 0.09$ & $0.591 \pm 0.015$ & $2.99 \pm 0.09$ \\
Conv. 1 ap & $0.563 \pm 0.005$ & $9.10 \pm 0.14$ & $0.596 \pm 0.015$ & $3.05 \pm 0.12$ \\
\hline Inv. no mask & $0.570 \pm 0.000$ & $10.99 \pm 0.12$ & $0.533 \pm 0.014$ & $3.34 \pm 0.08$ \\
Inv. 6 ap & $0.569 \pm 0.003$ & $10.11 \pm 0.14$ & $0.565 \pm 0.015$ & $3.25 \pm 0.06$ \\
Inv. 1 ap & $0.570 \pm 0.000$ & $9.69 \pm 0.10$ & $0.576 \pm 0.012$ & $3.18 \pm 0.06$ \\
\hline
\end{tabular}

Table 1. OPV device parameters measured under AM1.5G $100 \mathrm{~mW} \mathrm{~cm} \mathrm{c}^{-2}$ illumination for conventional and inverted devices with different aperture configurations. 


\begin{tabular}{|c|c|c|}
\hline Parameter & Conventional & Inverted \\
\hline P3HT:PCBM thickness [nm] & \multicolumn{2}{|c|}{250} \\
\hline Relative permittivity & \multicolumn{2}{|c|}{3.8} \\
\hline${ }^{\mathrm{a}}$ Electron affinity $[\mathrm{eV}]$ & \multicolumn{2}{|c|}{3.7} \\
\hline${ }^{\mathrm{b}}$ Effective bandgap [eV] & \multicolumn{2}{|c|}{1.1} \\
\hline${ }^{\mathrm{b}} \mathrm{CB} \& \mathrm{VB}$ effective density of states $\left[\mathrm{cm}^{-3}\right]$ & \multicolumn{2}{|c|}{$1 \times 10^{20}$} \\
\hline${ }^{\mathrm{b}}$ Anode work function $[\mathrm{eV}]$ & \multicolumn{2}{|c|}{4.5} \\
\hline${ }^{\mathrm{a}}$ Cathode work function $[\mathrm{eV}]$ & \multicolumn{2}{|c|}{4.0} \\
\hline${ }^{\mathrm{c}}$ Bimolecular recombination coefficient $\mathrm{k}\left[\mathrm{cm}^{3} \mathrm{~s}^{-1}\right]$ & \multicolumn{2}{|c|}{$2 \times 10^{-12}$} \\
\hline b,d Surface recombination velocity $\left[\mathrm{cm} \mathrm{s}^{-1}\right]^{2}$ & \multicolumn{2}{|c|}{$1 \times 10^{5}$} \\
\hline${ }^{\mathrm{c}}$ Electron mobility $\mu_{\mathrm{e}}\left[\mathrm{cm}^{2} \mathrm{~V}^{-1} \mathrm{~s}^{-1}\right]$ & \multicolumn{2}{|c|}{$2 \times 10^{-3}$} \\
\hline${ }^{\mathrm{c}}$ Hole mobility $\mu_{\mathrm{h}}\left[\mathrm{cm}^{2} \mathrm{~V}^{-1} \mathrm{~s}^{-1}\right]$ & & \\
\hline Dopant concentration $\mathrm{N}_{\mathrm{A}}\left[\mathrm{cm}^{-3}\right]$ & \multicolumn{2}{|c|}{$\begin{array}{l}5 \times 10^{-4} \\
7 \times 10^{15}\end{array}$} \\
\hline Series resistance $\left[\Omega \mathrm{cm}^{2}\right]$ & 2.9 & 2.7 \\
\hline Shunt resistance $\left[\Omega \mathrm{cm}^{2}\right]$ & $8.4 \times 10^{4}$ & $4.8 \times 10^{3}$ \\
\hline
\end{tabular}

${ }^{\mathrm{a}}$ From[42]

${ }^{\mathrm{b}}$ From[32]

'See Supplemental Data

${ }^{\mathrm{d}}$ Hole recombination velocity at active layer/ZnO EEL interface is $1 \times 10^{-5} \mathrm{~cm} / \mathrm{s}$

Table 2. Material parameters used for drift-diffusion simulation of conventional and inverted

OPVs. 


\begin{tabular}{lllll}
\hline Device & $\mathrm{V}_{\mathrm{os}}$ & $\begin{array}{l}\mathrm{J}_{\infty} \\
{\left[\mathrm{mA} \mathrm{cm}^{-2}\right]}\end{array}$ & FF & $\begin{array}{l}\text { PCE } \\
{[\%]}\end{array}$ \\
\hline Conv. & 0.524 & 11.57 & 0.587 & 3.56 \\
Inv. & 0.524 & 11.66 & 0.583 & 3.56 \\
\hline
\end{tabular}

Table 3. SCAPS simulated OPV device parameters under AM1.5G $100 \mathrm{~mW} \mathrm{~cm}^{-2}$ illumination for conventional and inverted devices. 


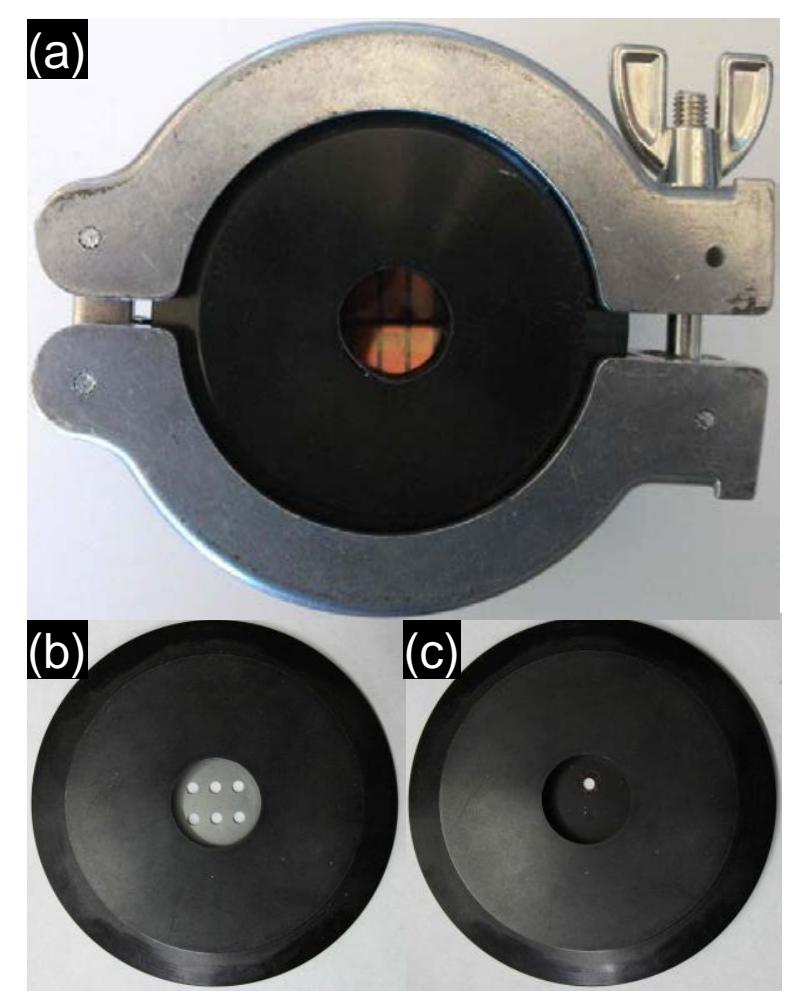

Fig. 1. (a) Assembled OPV holder with device and no mask. (b) Shadow mask with six $2.5 \mathrm{~mm}$ diameter apertures ("6 ap") in holder. (c) Shadow mask with one $2.5 \mathrm{~mm}$ diameter aperture ("1 ap") in holder. All views are from the solar simulator. 

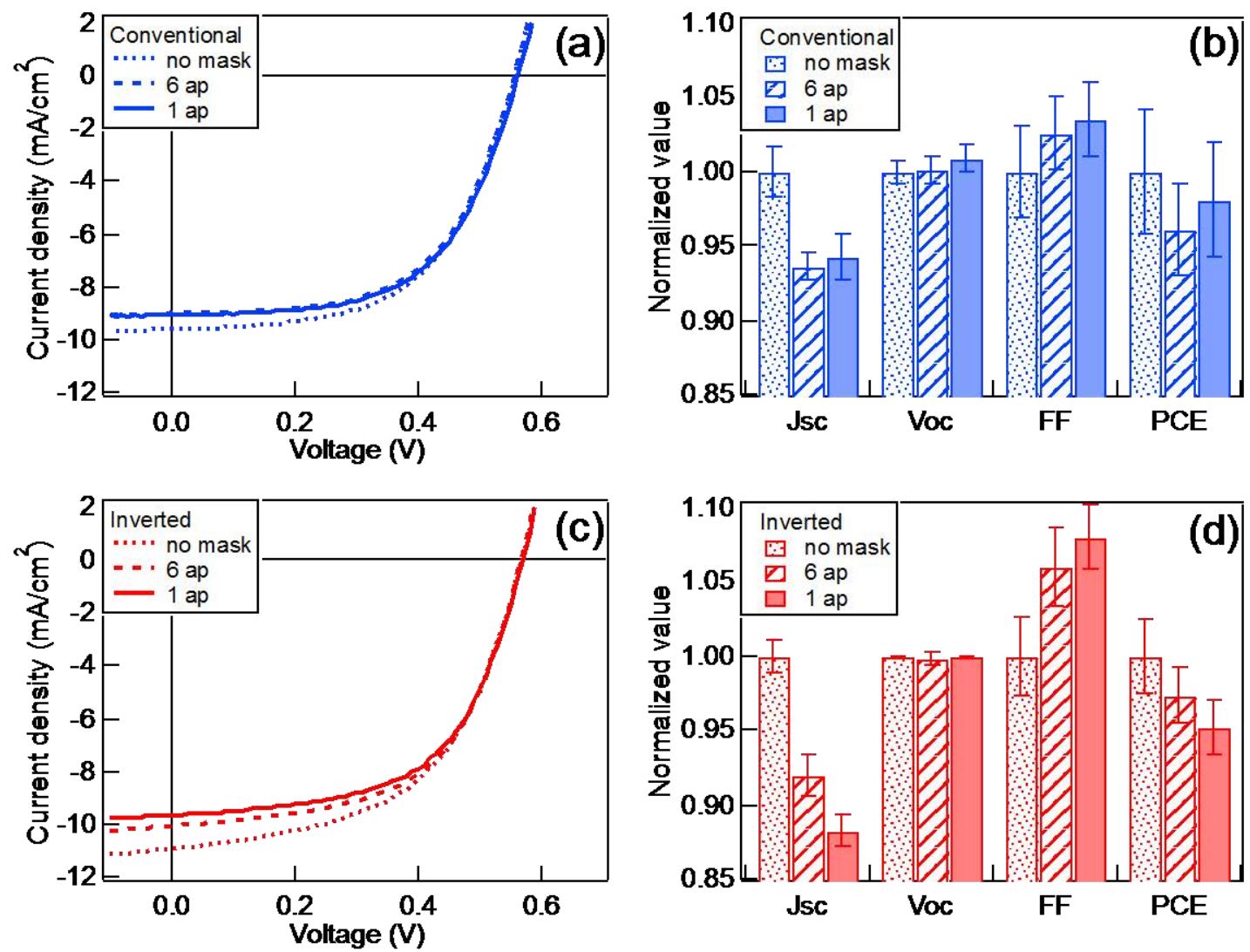

Fig. 2. (a) J-V under AM1.5G $100 \mathrm{~mW} \mathrm{~cm}{ }^{-2}$ of conventional OPVs with no mask (dotted), 6 ap mask (dashed), and 1 ap mask (solid). (b) OPV device parameters for conventional OPVs with no mask (dotted), 6 ap (striped), and 1 ap (solid) devices, normalized to the no mask device. (c) J-V under AM1.5G $100 \mathrm{~mW} \mathrm{~cm}^{-2}$ of inverted OPVs without mask (dotted), with 6 ap mask (dashed), and with 1 ap mask (solid). (d) OPV device parameters for conventional no mask (dotted), 6 ap (striped), and 1 ap (solid) devices, normalized to the no mask device. 

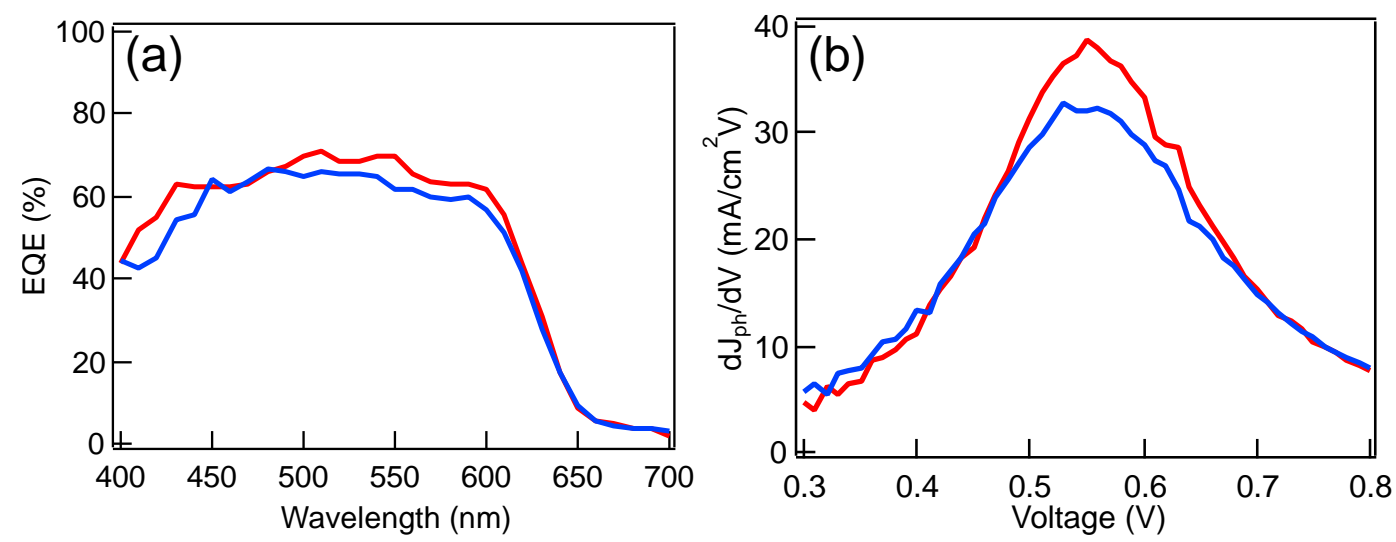

Fig. 3. (a) Experimental EQE spectra for conventional (blue) and inverted (red) OPVs. (b) $\mathrm{dJ}_{\mathrm{ph}} / \mathrm{dV}$ versus $\mathrm{V}$ for conventional (blue) and inverted (red) OPVs. 

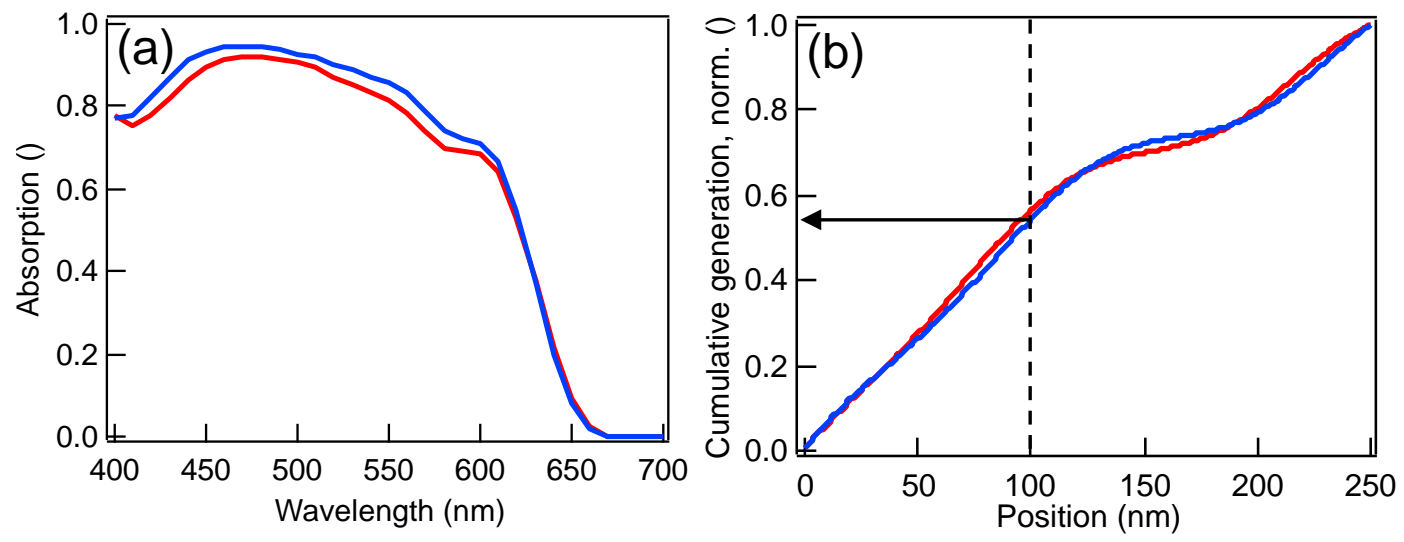

Fig. 4. (a) Fractional absorption spectra calculated using transfer matrix method for the active layer in conventional (blue) and inverted (red) OPVs. (b) Profiles of cumulative generation normalized to total generation, calculated from curves in Figure 4a for conventional (blue) and inverted (red) OPVs. 0-nm in the position corresponds to front EL/active layer interface. 

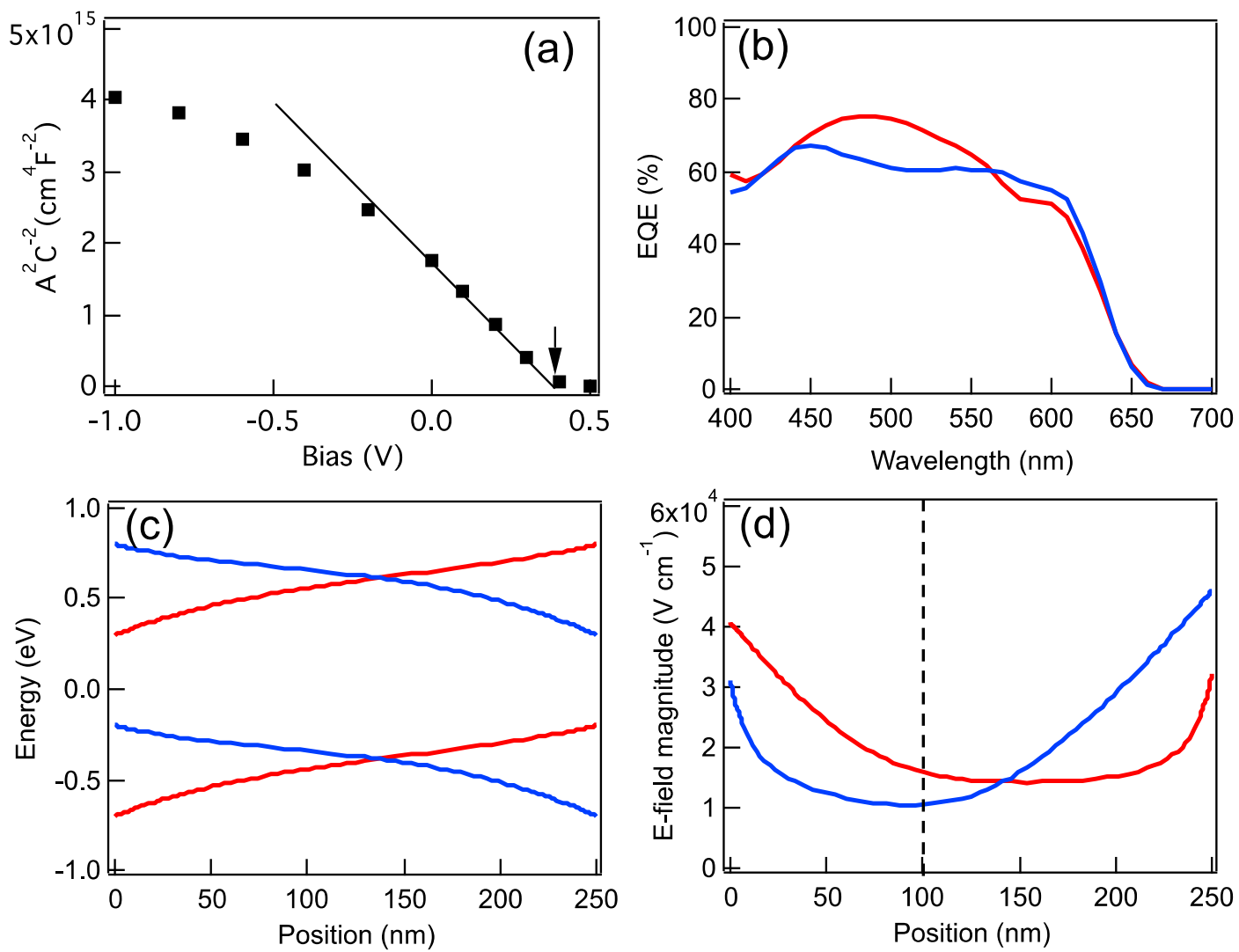

Fig. 5. (a) Mott-Schottky curve showing the $A^{2} C^{-2}$ (measured at $1 \mathrm{kHz}$ ) vs. V for a conventional OPV. Lines represent the fitting according to Eq. 1, from which $\mathrm{N}_{\mathrm{A}}$ of $7 \times 10^{15} \mathrm{~cm}^{-3}$ is obtained. Arrow indicates x-intercept voltage. (b) Simulated EQE spectra for conventional (blue) and inverted (red) OPVs. (c) Simulated band diagrams for conventional (blue) and inverted (red) OPVs at short circuit. (d) Simulated electric field magnitude for conventional (blue) and inverted (red) OPVs at short circuit. 0-nm in the position corresponds to front EL/active layer interface. 

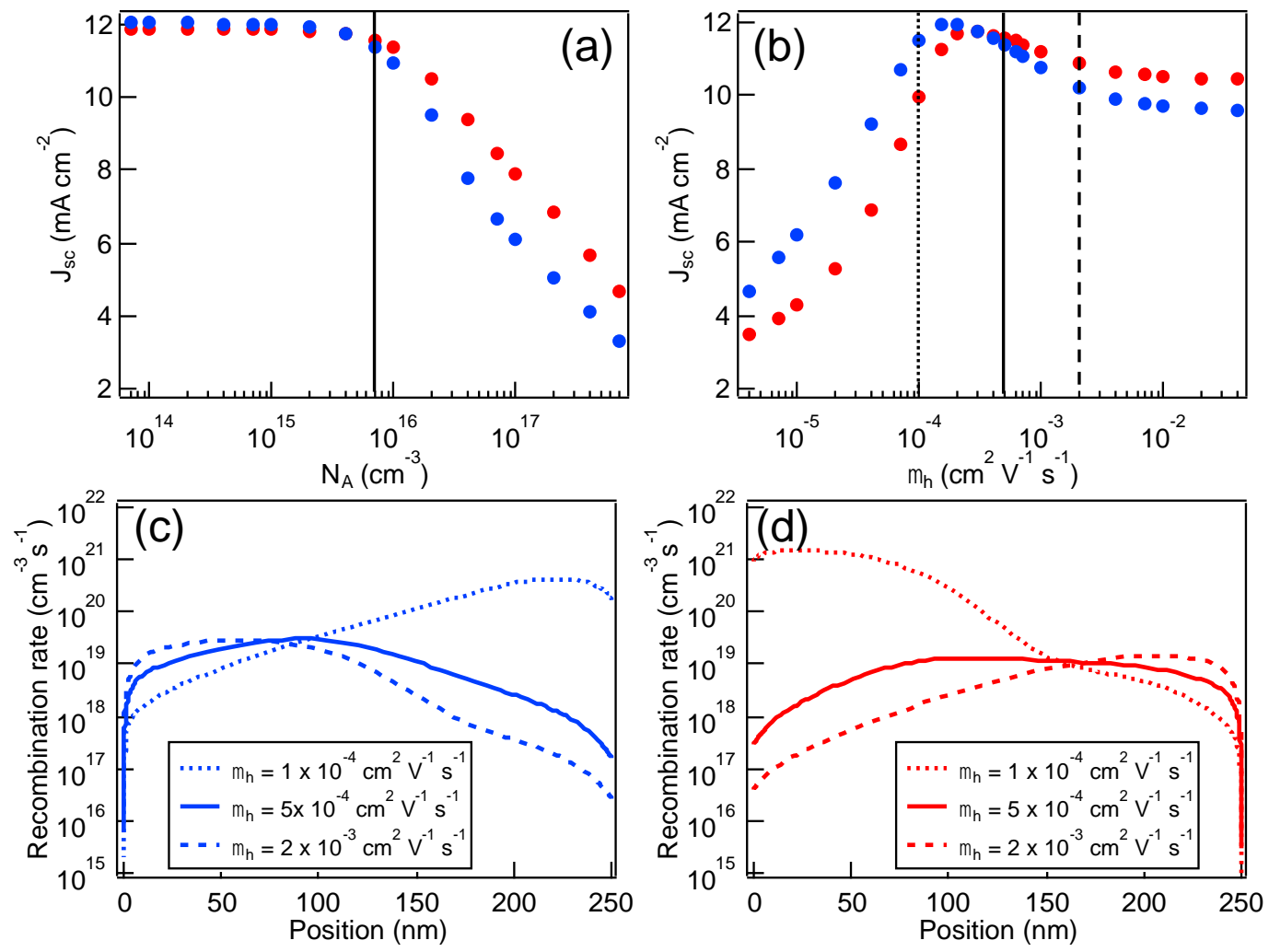

Fig. 6. Dependence of $J_{s c}$ for conventional (blue) and inverted (red) OPVs on (a) $N_{A}$ and (b) $\mu_{h}$.

Dashed lines correspond to values used in (c) and (d). Simulated recombination rate profiles for

(c) conventional and (d) inverted OPVs with $\mu_{\mathrm{h}}=1 \times 10^{-4} \mathrm{~cm}^{2} \mathrm{~V}^{-1} \mathrm{~s}^{-1}$ (dotted), $\mu_{\mathrm{h}}=5 \times 10^{-4} \mathrm{~cm}^{2}$ $\mathrm{V}^{-1} \mathrm{~s}^{-1}$ (solid), and $\mu_{\mathrm{h}}=2 \times 10^{-3} \mathrm{~cm}^{2} \mathrm{~V}^{-1} \mathrm{~s}^{-1}$ (dashed). 0 -nm in the position corresponds to front EL/active layer interface (HEL for (c) and EEL for (d)). 


\section{Reference}

[1] N. Li, D. Baran, K. Forberich, F. Machui, T. Ameri, et al., Towards 15\% energy conversion efficiency: a systematic study of the solution-processed organic tandem solar cells based on commercially available materials, Energy Environ. Sci. 6 (2013) 34073413. doi:10.1039/C3EE42307G.

[2] R.R. Sondergaard, M. Hösel, F.C. Krebs, Roll-to-roll fabrication of large area functional organic materials, J. Polym. Sci. Part B Polym. Phys. 51 (2013) 16-34. doi:10.1002/polb.23192.

[3] V. Shrotriya, G. Li, Y. Yao, T. Moriarty, K. Emery, Y. Yang, Accurate Measurement and Characterization of Organic Solar Cells, Adv. Funct. Mater. 16 (2006) 2016-2023. doi:10.1002/adfm.200600489.

[4] A. Cravino, P. Schilinsky, C.J. Brabec, Characterization of Organic Solar Cells: the Importance of Device Layout, Adv. Funct. Mater. 17 (2007) 3906-3910. doi:10.1002/adfm.200700295.

[5] D. Gupta, M. Bag, K.S. Narayan, Area dependent efficiency of organic solar cells, Appl. Phys. Lett. 93 (2008) 163301. doi:10.1063/1.2998540.

[6] C.-F. Lin, B.-H. Lin, S.-W. Liu, W.-F. Hsu, M. Zhang, et al., Optical effects of shadow masks on short circuit current of organic photovoltaic devices, Phys. Chem. Chem. Phys. 14 (2012) 3837-3842. doi:10.1039/c2cp00033d.

[7] H.J. Snaith, How should you measure your excitonic solar cells? Energy Environ. Sci. 5 (2012) 6513-6520. doi:10.1039/C2EE03429H.

[8] S.A. Gevorgyan, J.E. Carlé, R.R. Sondergaard, Accurate characterization of OPVs: Device masking and different solar simulators, Sol. Energ. Mat. Sol. Cells. 110 (2013) 24-35. doi:10.1016/j.solmat.2012.11.020.

[9] L.J. Rozanski, C.T.G. Smith, K.K. Gandhi, M.J. Beliatis, G.D.M.R. Dabera, et al., A critical look at organic photovoltaic fabrication methodology: Defining performance enhancement parameters relative to active area, Sol. Energ. Mat. Sol. Cells. 130 (2014) 513-520. doi:10.1016/j.solmat.2014.07.054.

[10] S.A. Gevorgyan, M. Jorgensen, F.C. Krebs, A setup for studying stability and degradation of polymer solar cells, Sol. Energ. Mat. Sol. Cells. 92 (2008) 736-745. doi:10.1016/j.solmat.2008.02.008.

[11] M.O. Reese, A.K. Sigdel, J.J. Berry, D.S. Ginley, S.E. Shaheen, A simple miniature controlled-atmosphere chamber for optoelectronic characterizations, Sol. Energ. Mat. Sol. Cells. 94 (2010) 1254-1258. doi:10.1016/j.solmat.2010.03.017.

[12] S.A. Gevorgyan, M. Jorgensen, F.C. Krebs, K.O. Sylvester-Hvid, A compact multichamber setup for degradation and lifetime studies of organic solar cells, Sol. Energ. Mat. Sol. Cells. 95 (2011) 1389-1397. doi:10.1016/j.solmat.2011.01.023.

[13] R. Steim, F.R. Kogler, C.J. Brabec, Interface materials for organic solar cells, J. Mater. Chem. 20 (2010) 2499-2512. doi:10.1039/b921624c.

[14] H.-L. Yip, A.K.Y. Jen, Recent advances in solution-processed interfacial materials for efficient and stable polymer solar cells, Energy Environ. Sci. 5 (2012) 5994-6011. doi:10.1039/c2ee02806a.

[15] Z. Xu, L.-M. Chen, G. Yang, C.-H. Huang, J. Hou, et al., Vertical Phase Separation in Poly(3-hexylthiophene): Fullerene Derivative Blends and its Advantage for Inverted Structure Solar Cells, Adv. Funct. Mater. 19 (2009) 1227-1234. 
doi:10.1002/adfm.200801286.

[16] D.S. Germack, C.K. Chan, R.J. Kline, D.A. Fischer, D.J. Gundlach, et al., Interfacial Segregation in Polymer/Fullerene Blend Films for Photovoltaic Devices, Macromolecules. 43 (2010) 3828-3836. doi:10.1021/ma100027b.

[17] E. Pavlopoulou, G. Fleury, D. Deribew, F. Cousin, Phase separation-driven stratification in conventional and inverted P3HT:PCBM organic solar cells, Org. Electron. 14 (2013) 1249-1254. doi:10.1016/j.orgel.2013.02.020.

[18] J.R. Tumbleston, D.-H. Ko, E.T. Samulski, R. Lopez, Analyzing local exciton generation profiles as a means to extract transport lengths in organic solar cells, Phys. Rev. B. 82 (2010) 205325. doi:10.1103/PhysRevB.82.205325.

[19] T.J.K. Brenner, Hwang, Inchan, N.C. Greenham, C.R. McNeill, Device physics of inverted all-polymer solar cells, J. Appl. Phys. 107 (2010) 114501. doi:10.1063/1.3371364.

[20] T.J.K. Brenner, Y. Vaynzof, Z. Li, D. Kabra, R.H. Friend, C.R. McNeill, White-light bias external quantum efficiency measurements of standard and inverted P3HT : PCBM photovoltaic cells, J. Phys. D Appl. Phys. 45 (2012) 415101. doi:10.1088/00223727/45/41/415101.

[21] S.R. Cowan, J. Wang, J. Yi, Y.-J. Lee, D.C. Olson, J.W.P. Hsu, Intensity and wavelength dependence of bimolecular recombination in P3HT:PCBM solar cells: A white-light biased external quantum efficiency study, J. Appl. Phys. 113 (2013) 154504. doi:10.1063/1.4801920.

[22] I. Etxebarria, A. Guerrero, J. Albero, G. Garcia-Belmonte, E. Palomares, R. Pacios, Inverted vs standard PTB7:PC70BM organic photovoltaic devices. The benefit of highly selective and extracting contacts in device performance, Org. Electron. 15 (2014) 27562762. doi:10.1016/j.orgel.2014.08.008.

[23] T. Ameri, G. Dennler, C. Waldauf, P. Denk, Realization, characterization, and optical modeling of inverted bulk-heterojunction organic solar cells, J. Appl. Phys. 103 (2008) 084506. doi:10.1063/1.2902804.

[24] D. Chen, C. Zhang, Z. Wang, J. Zhang, Q. Feng, et al., Performance Comparison of Conventional and Inverted Organic Bulk Heterojunction Solar Cells From Optical and Electrical Aspects, IEEE Trans. Electron Devices. 60 (2013) 451-457. doi:10.1109/TED.2012.2224114.

[25] C.E. Petoukhoff, D.K. Vijapurapu, D.M. O'Carroll, Computational comparison of conventional and inverted organic photovoltaic performance parameters with varying metal electrode surface workfunction, Sol. Energ. Mat. Sol. Cells. 120 (2014) 572-583. doi:10.1016/j.solmat.2013.09.041.

[26] J. Wang, Y.-J. Lee, J.W.P. Hsu, One-step synthesis of ZnO nanocrystals in n-butanol with bandgap control: applications in hybrid and organic photovoltaic devices, J. Phys. Chem. C. 118 (2014) 18417-18423. doi:10.1021/jp505058u.

[27] G.F. Burkhard, E.T. Hoke, M.D. McGehee, Accounting for Interference, Scattering, and Electrode Absorption to Make Accurate Internal Quantum Efficiency Measurements in Organic and Other Thin Solar Cells, Adv. Mater. 22 (2010) 3293-3297. doi:10.1002/adma.201000883.

[28] M. Burgelman, P. Nollet, S. Degrave, Modelling polycrystalline semiconductor solar cells, Thin Solid Films. 361-362 (2000) 527-532. doi:10.1016/S0040-6090(99)00825-1.

[29] M. Mingebach, C. Deibel, V. Dyakonov, Built-in potential and validity of the Mott- 
Schottky analysis in organic bulk heterojunction solar cells, Phys. Rev. B. 84 (2011) 153201. doi:10.1103/PhysRevB.84.153201.

[30] B.A. Gregg, Charged defects in soft semiconductors and their influence on organic photovoltaics, Soft Matter. 5 (2009) 2985. doi:10.1039/b905722f.

[31] G. Garcia-Belmonte, A. Munar, E.M. Barea, J. Bisquert, I. Ugarte, R. Pacios, Charge carrier mobility and lifetime of organic bulk heterojunctions analyzed by impedance spectroscopy, Org. Electron. 9 (2008) 847-851. doi:10.1016/j.orgel.2008.06.007.

[32] T. Kirchartz, W. Gong, S.A. Hawks, T. Agostinelli, R.C.I. MacKenzie, et al., Sensitivity of the Mott-Schottky Analysis in Organic Solar Cells, J. Phys. Chem. C. 116 (2012) 7672-7680. doi:10.1021/jp300397f.

[33] J.C. Blakesley, F.A. Castro, W. Kylberg, G.F.A. Dibb, C. Arantes, et al., Towards reliable charge-mobility benchmark measurements for organic semiconductors, Org. Electron. 15 (2014) 1263-1272. doi:10.1016/j.orgel.2014.02.008.

[34] A.J. Morfa, A.M. Nardes, S.E. Shaheen, N. Kopidakis, J. van de Lagemaat, Time-ofFlight Studies of Electron-Collection Kinetics in Polymer:Fullerene Bulk-Heterojunction Solar Cells, Adv. Funct. Mater. 21 (2011) 2580-2586. doi:10.1002/adfm.201100432.

[35] V.A. Trukhanov, V.V. Bruevich, D.Y. Paraschuk, Effect of doping on performance of organic solar cells, Phys. Rev. B. 84 (2011) 205318. doi:10.1103/PhysRevB.84.205318.

[36] T. Kirchartz, T. Agostinelli, M. Campoy-Quiles, W. Gong, J. Nelson, Understanding the Thickness-Dependent Performance of Organic Bulk Heterojunction Solar Cells: The Influence of Mobility, Lifetime, and Space Charge, J. Phys. Chem. Lett. 3 (2012) 34703475. doi:10.1021/jz301639y.

[37] B. Lüssem, M. Riede, K. Leo, Doping of organic semiconductors, Phys. Status Solidi A. 210 (2013) 9-43. doi:10.1002/pssa.201228310.

[38] G.F.A. Dibb, M.-A. Muth, T. Kirchartz, S. Engmann, H. Hoppe, et al., Influence of doping on charge carrier collection in normal and inverted geometry polymer:fullerene solar cells, Sci. Rep. 3 (2013). doi:10.1038/srep03335.

[39] F. Deledalle, T. Kirchartz, M.S. Vezie, M. Campoy-Quiles, P.S. Tuladhar, et al., Understanding the Effect of Unintentional Doping on Transport Optimization and Analysis in Efficient Organic Bulk-Heterojunction Solar Cells, Phys. Rev. X. 5 (2015) 011032. doi:10.1103/PhysRevX.5.011032.

[40] J.A. Bartelt, D. Lam, T.M. Burke, S.M. Sweetnam, M.D. McGehee, Charge-Carrier Mobility Requirements for Bulk Heterojunction Solar Cells with High Fill Factor and External Quantum Efficiency $>90 \%$, Adv. Energy Mater. (2015) n/a-n/a. doi:10.1002/aenm.201500577.

[41] V. Mihailetchi, J. Wildeman, P. Blom, Space-Charge Limited Photocurrent, Phys. Rev. Lett. 94 (2005) 126602. doi:10.1103/PhysRevLett.94.126602.

[42] B.W. Larson, J.B. Whitaker, X.-B. Wang, A.A. Popov, G. Rumbles, et al., Electron Affinity of Phenyl-C61-Butyric Acid Methyl Ester (PCBM), J. Phys. Chem. C. 117 (2013) 14958-14964. doi:10.1021/jp403312g. 


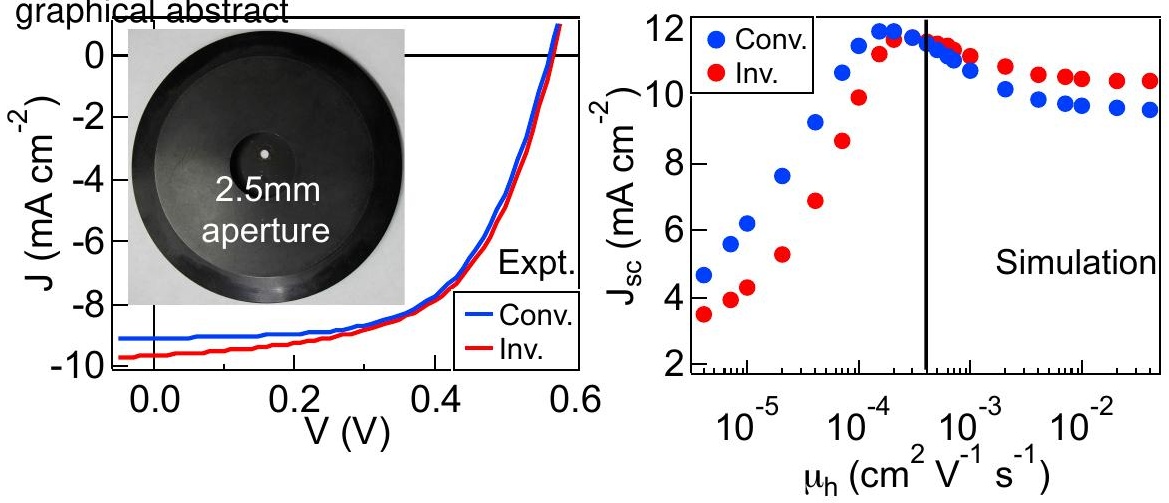

